\section{Espacios 100\% libres de humo de tabaco en la UAZ, como prevención de la Epidemia del Tabaquismo}

\section{Resumen}

Introducción: La epidemia mundial del tabaquismo mata cada año a casi 6 millones de personas, de las cuales más de 600000 son no fumadores que mueren por respirar humo ajeno. Si no actuamos, la epidemia matará a más de 8 millones de personas al año de aquí a 2030. Más del $80 \%$ de esas muertes evitables se producirán entre las poblaciones de los países de ingresos bajos y medianos (Organización Mundial de la Salud 2014).

De ahí la importancia de promover espacios 100\% libres de humo de tabaco, esta acción entre los jóvenes que son la población susceptible. En la UAZ con la implementación del Modelo académico UAZ Siglo $X X I$, contempla la formación integral de nuestros alumnos que conlleva a un desarrollo sostenible que sea para toda la vida.

Objetivo: Implementar en todos los espacios Universitarios, espacios $100 \%$ libres de humo de tabaco.

Material y Métodos: El proceso se llevó en 2 etapas: (2007-2010), (2011-2013). Participando todos los sectores que conforman la UAZ, alumnos, docentes, trabajadores, autoridades administrativas. Procedimiento: 1.-Solicitud a la Secretaría de Salud, 2.- Que realizó cinco visitas, 3.-Que la Comunidad estuviera enterada del proceso, 4.-Colocación de ceniceros a la entrada del edificio, 5.-Señalamientos de espacio 100\% libre de Humo de Tabaco, 6.-Señalamiento del Reglamento de la UAZ como espacio 100\% libre de Humo de Tabaco.

Resultados: La primera Etapa, se certificó el área de Ciencias de la Salud y CECIUAZ. En la segunda etapa se certificó 45 espacios de la UAZ. Conclusiones: Con este reconocimiento inicia el trabajo de retroalimentación y forma parte de la formación integral de nuestros alumnos, y del personal docente, trabajadores y administración central. Se programa una evaluación del programa en dos años.

Palabras claves: Espacios 100\% libres de humo de tabaco. UAZ.
Moreno García María Alejandra, Chávez Ruvalcaba María Isabel, Maldonado Tapia Claudia, Muñoz Moreno Claudia Yadira, Rivas Gutiérrez Jesús, Muñoz Escobedo José Jesús

Universidad Autónoma de Zacatecas. México. Cuerpo Académico de Biología Celular y Microbiología C-103

Correspondencia:

”amoreno_29@hotmail.com 


\section{Introducción}

Según el Atlas Mundial del Tabaco (Eriksen, Mackay y Ross, 2012), la industria tabacalera es millonaria y de 2000 a 2010, su producción aumentó $12 \%$, fabricando cerca de 6 quintillones de cigarros al año, aproximadamente un millón cada cinco minutos. En 2010, el ingreso total consolidado de las seis principales compañías tabacaleras en el mundo (que incluye a Phillip Morris y a British American Tabaco) (INEGI 2013).

El tabaco cuesta a la economía mundial 200 mil millones de dólares cada año.

En 2010, la población adulta mexicana (de 18 a 65 años) gasta en promedio 6 días de salario mínimo al mes en tabaco. De acuerdo con la Encuesta Nacional de Adicciones 2011, casi la mitad de los fumadores iniciaron el consumo antes de los 16 años.

En 2012, la prevalencia de consumo actual de tabaco es más alta en hombres que en mujeres (31\% en hombres y 9\% mujeres). Durante 2011 en México, 29 de cada 100 muertes se deben a enfermedades relacionadas con el tabaco.

En 2011, la población mexicana muere principalmente por enfermedades isquémicas del corazón, $45.3 \%$ en los hombres y $42.3 \%$ en las mujeres. (INEGI 2013).

Investigaciones relacionadas con los efectos del tabaco en el cuerpo han identificado que el factor adictivo a la nicotina es similar al del alcohol, la cocaína y la morfina (Medline, 2013); además, consumir tabaco aumenta el riesgo de padecer cáncer especialmente en pulmones, boca, laringe, esófago, vejiga, riñón, páncreas y cuello del útero-, ataques cardíacos y apoplejías, y enfermedad pulmonar crónica (Medline, 2013).

Las consecuencias de su consumo no se limitan al fumador, las personas que están expuestas al humo del tabaco tienen más riesgos de sufrir enfermedades coronarias y cáncer de pulmón. En los niños puede haber enfermedades del oído me- dio, síntomas respiratorios, limitación de la función respiratoria, síndrome infantil de muerte de cuna, enfermedades de las vías respiratorias bajas y bajo peso al nacer (Eriksen, Mackay, y Ross, 2012).

En las mujeres embarazadas, el contacto con el humo del tabaco aumenta el riesgo de placenta previa, ruptura prematura de membranas, parto prematuro, aborto espontáneo y embarazo ectópico; mientras que en el producto se afecta el desarrollo fetal, se reduce la función pulmonar, presenta cáncer oral, e incluso pueden llegar a muerte fetal -óbito- o síndrome de muerte súbita del infante (Eriksen, Mackay, y Ross, 2012).

Las secuelas de esta adicción son múltiples, por eso las ciencias médicas y de la conducta aplican tratamientos para mejorar la calidad de vida de las personas que las sufren. Sin embargo, para lograr cambiar este hábito es importante el autocontrol, la motivación al cambio y las habilidades de afrontamiento (Froján y Becoña, 1999) y cuando se logra, dar seguimiento a los pacientes, para evitar recaídas que se asocian a estados emocionales negativos, altos niveles de dependencia y escaso grado de apoyo social (Quesada, Carreras y Sánchez, 2002).

\section{Convenio Marco de la OMS para el Control del Tabaco}

La idea de crear un instrumento internacional para el control del tabaco surgió a raíz de la adopción de la resolución WHA48.11, en mayo de 1995, mediante la cual se pedía al Director General que informara a la 49a Asamblea Mundial de la Salud sobre la viabilidad de elaborar un instrumento internacional, por ejemplo directrices, una declaración, o un convenio internacional sobre lucha antitabáquica.

El Convenio Marco de la OMS para el Control del Tabaco (CMCT OMS) es el primer tratado negociado bajo los auspicios de la Organización Mundial de 
la Salud. EI CMCT OMS es un tratado basado en pruebas científicas que reafirma el derecho de todas las personas a gozar del grado máximo de salud que se pueda lograr. El CMCT OMS representa un cambio ejemplar en el desarrollo de una estrategia normativa para abordar las cuestiones relativas a las sustancias adictivas; a diferencia de anteriores tratados sobre fiscalización de drogas, el CMCT OMS afirma la importancia de las estrategias de reducción de la demanda, así como de ciertas cuestiones relativas al suministro.

EI CMCT OMS se elaboró en respuesta a la globalización de la epidemia de tabaquismo.

La propagación de esa epidemia se ve favorecida por diversos factores complejos con efectos transfronterizos, entre ellos la liberalización del comercio y las inversiones extranjeras directas.

Otros factores tales como la comercialización a nivel mundial, la publicidad transnacional del tabaco, la promoción y el patrocinio, así como el tráfico internacional de cigarrillos de contrabando y falsificados también han contribuido al espectacular aumento del tabaquismo.

Medidas relacionadas con los precios e impuestos para reducir la demanda de tabaco, y medidas no relacionadas con los precios para reducir la demanda de tabaco, a saber: protección contra la exposición al humo de tabaco, reglamentación del contenido de los productos de tabaco, reglamentación de la divulgación de información sobre los productos de tabaco, empaquetado y etiquetado de los productos de tabaco, educación, comunicación, formación y concientización del público, publicidad, promoción y patrocinio del tabaco, y medidas de reducción de la demanda relativas a la dependencia y al abandono del tabaco.

Tras su adopción por la 56 Asamblea Mundial de la Salud, en mayo de 2003, el Convenio Marco de la OMS para el Control del Tabaco (CMCT de la OMS) estuvo abierto a la firma hasta el 29 de junio de 2004. Durante ese periodo, 168 Estados suscribieron el CMCT de la OMS, manifestando su intención de ser Partes en el Convenio. Los países que desean ser Partes en el Convenio, pero que no estuvieron en condiciones de firmarlo dentro del plazo que finalizó el 29 de junio de 2004, pueden hacerlo mediante la adhesión, que es un proceso de una sola fase equivalente a la ratificación.

De conformidad con el artículo 36 del CMCT de la OMS, el Convenio entró en vigor el 27 de febrero de 2005, es decir, al nonagésimo día contado desde la fecha de adhesión, ratificación, aceptación o aprobación por el cuadragésimo Estado. Está depositado en la Sede de las Naciones Unidas, depositaria del tratado, en Nueva York, y continúa abierto a los Miembros de la Organización Mundial de la Salud (OMS) y a los Estados que no son miembros de la OMS pero sí lo son de las Naciones Unidas, así como a las organizaciones de integración económica regional (Convenio marco de la OMS para el control del tabaco. 2010).

Este Convenio entró en vigor el 27 de febrero de 2005 para los primeros 40 países que lo ratificaron, entre los cuales México fue el primer país de Américas en adherirse.

De conformidad con el CMCT/OMS, son cuatro los pilares en los que se sustentan las políticas públicas para la prevención y el combate a la epidemia del tabaquismo:

1. Promoción de espacios $100 \%$ libres de humo de tabaco.

2. Prohibición total de toda forma de publicidad, patrocinio y promoción.

3. Incorporación de advertencias sanitarias y pictogramas a todo empaquetado y etiquetado de los productos del tabaco.

4. Política fiscal para disminuir la demanda de productos de tabaco, principalmente entre los jóvenes y otros grupos vulnerables (Página de la Organización Mundial de la Salud 2013). 


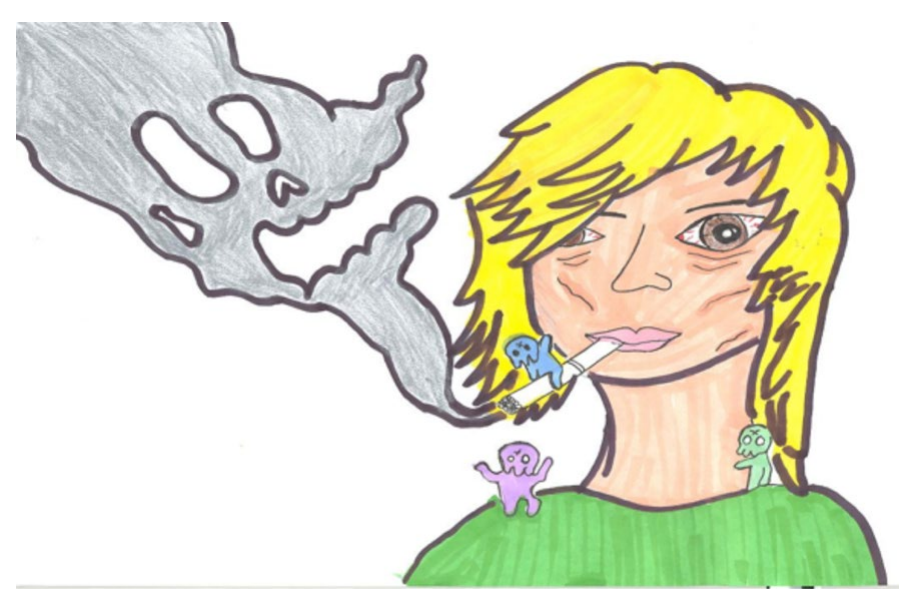

Figura 1. Pictograma en la campaña antitabaco UAZ, el humo del tabaco causante de morbilidad y mortalidad en el ser humano en fumadores pasivos y activos (Dibujo Claudia Muñoz M-2007)

\section{Elementos clave del Tratado}

Entre muchas otras medidas, el tratado exige que los países impongan restricciones a la publicidad, el patrocinio y la promoción del tabaco; adopten un nuevo envasado y etiquetado de los productos de tabaco; establezcan controles para velar por un aire limpio en ambientes interiores; y fortalezcan la legislación para combatir enérgicamente el contrabando de tabaco.

\section{Publicidad, patrocinio y promoción}

Se publicitan productos de tabaco en competiciones deportivas, conciertos y películas, así como a través de la moda $y$, en realidad, en cualquier lugar desde el cual la industria tabacalera pueda dirigirse a nuevos fumadores potenciales. El tratado obliga a los Estados Partes a proceder a una prohibición integral de la publicidad, la promoción y el patrocinio del tabaco, en la medida en que lo permitan sus respectivas constituciones. Las Partes cuya constitución o principios constitucionales no permitan una prohibición integral deben aplicar una serie de restricciones a toda publicidad, promoción y patrocinio.

\section{Envasado y etiquetado de los productos de tabaco}

A medida que se aplican las restricciones a la publicidad, el envasado del tabaco desempeña una función cada vez más importante en el consumo del producto. El tratado obliga a los Estados Partes a adoptar e introducir en los productos de tabaco y el envasado externo de los mismos advertencias y mensajes sanitarios grandes, claros, visibles, legibles y rotativos que ocupen al menos el 30\% de la superficie externa principal. Esto se exige dentro de un plazo de tres años a partir de la entrada en vigor del Convenio.

\section{Protección de la exposición al humo de tabaco}

El humo de tabaco de segunda mano es una amenaza real y considerable para la salud pública. Los niños corren riesgos particulares; la exposición de los niños al humo de tabaco puede causar afecciones respiratorias, enfermedades del oído medio, ataques de asma y síndrome de muerte súbita del recién nacido (SMSRN). El tratado obliga a los Estados Partes a adoptar y aplicar (en las áreas sometidas a la jurisdicción nacional vigente y de conformidad con la legislación nacional o promover (en 


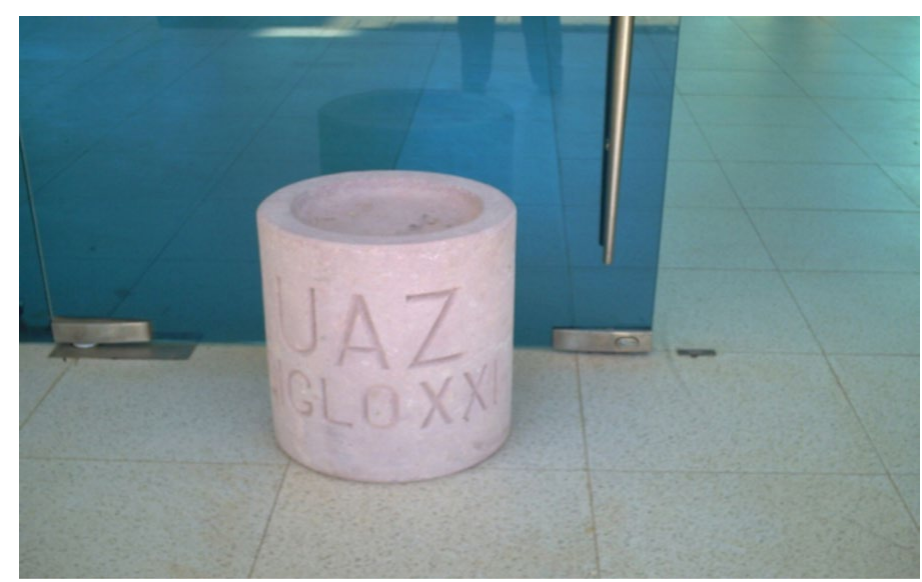

Figura 2. Cenicero colocado en la puerta del Edificio 1 del Área de Ciencias Salud UAZ. 2008.

otros niveles jurisdiccionales) medidas eficaces que protejan de la exposición al humo de tabaco en lugares de trabajo interiores, transportes públicos, lugares públicos interiores y, según convenga, otros lugares públicos.

\section{Comercio ilícito de productos de tabaco}

Los cigarrillos son objeto frecuente de contrabando en todo el mundo. Además de conseguir que las marcas internacionales sean más asequibles y accesibles, los cigarrillos que son objeto de comercio ilegal evaden las restricciones y la reglamentación sanitaria. El tratado obliga a los Estados Partes a adoptar y aplicar medidas eficaces para eliminar el comercio ilícito, la fabricación ilícita y la falsificación de productos de tabaco.

\section{Problemática}

Recientemente México aprobó una ley federal (2008 y 2010) y otra en la capital del país (2008) que prohíben fumar en espacios cerrados. Estas medidas legislativas están en plena concordancia con las acciones propuestas en el Artículo 8 del Convenio Marco para el Control del Tabaco de la Organización Mundial de la Salud, que México ratificó en 2004.
En este ensayo se revisa la evidencia científica que demuestra que la exposición al humo de tabaco causa enfermedades crónicas y agudas en niños y adultos no fumadores. Toda exposición al humo de tabaco es dañina, por lo tanto sólo la creación de espacios 100\% libres de humo de tabaco, tal como lo plantean las nuevas leyes, constituye una intervención efectiva para proteger a la población.

\section{Metodología}

El proceso se llevó en 2 etapas: (2007-2010), (2011-2013).

Participando todos los sectores que conforman la UAZ, alumnos, docentes, trabajadores, autoridades administrativas.

Comunicado de las autoridades universitarias: Rector, Secretaria General, Secretaria Académica, Secretaria administrativa, Directores de todas las unidades, informando a la comunidad universitaria del proceso.

\section{Estimada comunidad Universitaria (2007-} 2013).

El 31 de mayo de cada año se celebra el día mundial contra el tabaco, en esta ocasión la Universidad Autónoma de Zacatecas, adquirió el compromiso 
de certificarse como espacio $100 \%$ libre de humo de tabaco.

Para lograr el cumplimiento de esta meta, es indispensable la colaboración de todos los universitarios: Alumnos, Trabajadores y Docente.

Esta planteada la siguiente ruta de trabajo.

1. Informar a todos los Universitarios, que se iniciaran los trabajos para ser certificados los espacios de la UAZ como $100 \%$ libres de humo de tabaco.

2. Que como norma de la Secretaria de Salud no debe existir ningún cenicero en los interiores de los espacios Universitarios.

3. Que la Institución colocara los señalamientos en lugares estratégicos como espacio 100\% libre de humo de tabaco.

4. Se colocara en las entradas principales ceniceros para depositar los residuos de tabaco antes de entrar a los edificios Universitarios.

5. En lugar estratégico a la entrada de cada espacio Universitario el reglamento de la UAZ como espacio $100 \%$ libre de humo de tabaco.

6. La meta es que en el 2013 todos los espacios de la UAZ sean certificados.

7. Te invitamos a formar parte de las brigadas de apoyo de cada espacio como vigilantes para mantener los espacios $100 \%$ libres de humo de tabaco.

\section{Procedimiento}

1. Solicitud a la Secretaría de Salud al director general de los servicios de salud del estado de Zacatecas por el Sr. Rector de la UAZ (esta acción se realizo en 12 etapas).

2. Cinco visitas Secretaria de salud.

Aplicando el siguiente cuestionario (Tabla 1).

Procedimiento de la Dirección General Técnica en adicciones y salud mental: procedimiento para el reconocimiento de edificios libres de humo de tabaco.

\section{Objetivo}

Reconocer aquellos inmuebles, donde existan evidencias incuestionables tanto de la ausencia del acto de fumar en las instalaciones como de protección a los no fumadores, como "EDIFICIOS LIBRES DE HUMO DE TABACO", a través de la observación dirigida y el interrogatorio a informantes clave.

\section{Procedimiento}

Visitar el edificio en cinco ocasiones, de las cuales la primera visita es anunciada y las cuatro sucesivas se realizan en forma aleatoria y sin anuncio previo.

Se aplica cada visita la cédula para ello preparada, obteniendo cinco calificaciones totales las cuales tendrán que ser promediadas para obtener la cédula matriz del procedimiento.

En caso de que en alguna visita el ítem 8 sea respondido en forma afirmativa, el procedimiento es cancelado, declarando al edificio como No Libre de Humo de Tabaco.

Existen ítems que siendo afirmativa la respuesta en la primera visita, lo serán seguramente en las sucesivas por lo que podrían obviarse su observación en estas últimas, tal es el caso de los ítems $\mathbf{1 , 2}, \mathbf{6}$, 7, 9, 10, 11, 12.

Los ítems 3 y 4 corresponden al conocimiento del programa por parte de empleados, tanto directivos y mandos medios como personal en general, para lograr una respuesta afirmativa de cada ítem se deberá interrogar en cada visita y en forma aleatoria a un directivo, tres mandos medios y cinco trabajadores en general, asegurándose que en cada visita las personas entrevistadas sean distintas.

Para lograr una respuesta afirmativa en el ítem tres, por lo menos cuatro trabajadores deberán responder positivamente en cada visita.

Para lograr una respuesta afirmativa en el ítem cuatro, el directivo tendrá que hacerlo en forma positiva y por lo menos dos de los tres mandos medios en cada ocasión.

Posterior al saludo y a su presentación como personal de salud y antes de comentar el objetivo de 
Tabla 1. Criterios para el reconocimiento de edificios libres de humo de tabaco

\begin{tabular}{|c|c|c|c|c|c|c|}
\hline \multirow{2}{*}{ No. } & \multirow{2}{*}{ Especificación } & \multirow{2}{*}{ Elementos de sustento } & \multirow{2}{*}{ Sitio } & \multicolumn{2}{|c|}{ Respuesta } & \multirow{2}{*}{ Cal. } \\
\hline & & & & Si & No & \\
\hline 1 & $\begin{array}{l}\text { ¿Existe en el edificio algún programa para la } \\
\text { protección de los no fumadores? }\end{array}$ & Documentos respectivos & $\begin{array}{l}\text { Dirección de } \\
\text { Administración }\end{array}$ & 2 & 0 & \\
\hline 2 & ¿Existe difusión de dicho programa? & $\begin{array}{l}\text { Periódico mural, folletos, } \\
\text { anuncios y otros medios }\end{array}$ & $\begin{array}{l}\text { Oficinas, pasillos } \\
\text { y otros sitios de } \\
\text { reunión }\end{array}$ & 2 & 0 & \\
\hline 3 & $\begin{array}{l}\text { ¿Conocen los empleados el programa en } \\
\text { cuestión? }\end{array}$ & Entrevista aleatoria & $\begin{array}{l}\text { Oficinas, salas de } \\
\text { junta, etc. }\end{array}$ & 2 & 0 & \\
\hline 4 & $\begin{array}{l}\text { ¿Conocen los directivos y mandos medios } \\
\text { dicho programa? }\end{array}$ & Entrevista aleatoria & $\begin{array}{l}\text { Oficinas, salas de } \\
\text { junta, etc. }\end{array}$ & 2 & 0 & \\
\hline 5 & $\begin{array}{l}\text { ¿Sabe el público usuario o visitante que no } \\
\text { se puede fumar dentro del edificio? }\end{array}$ & Entrevista aleatoria & $\begin{array}{l}\text { Salas de espera, } \\
\text { pasillos, etc. }\end{array}$ & 1 & 0 & \\
\hline 6 & $\begin{array}{l}\text { ¿Existe la leyenda "Por favor, apague su } \\
\text { cigarrillo antes de entrar" o alguna similar } \\
\text { en la entrada del edificio? }\end{array}$ & Observación & $\begin{array}{l}\text { Entrada del } \\
\text { edificio }\end{array}$ & 2 & 0 & \\
\hline 7 & $\begin{array}{l}\text { ¿Existen avisos o símbolos visuales que } \\
\text { expresen la prohibición de fumar? }\end{array}$ & Observación & $\begin{array}{l}\text { Áreas comunes } \\
\text { y pasillos } \\
\text { principales }\end{array}$ & 5 & 0 & \\
\hline 8 & $\begin{array}{l}\text { ¿Existen en el edificio indicios de uso } \\
\text { de tabaco (ceniceros, cajetillas, colillas, } \\
\text { cenizas, olor a humo de tabaco o personas } \\
\text { fumando? }\end{array}$ & Observación & $\begin{array}{l}\text { Oficinas, baños, } \\
\text { áreas comunes }\end{array}$ & 0 & 5 & \\
\hline 9 & $\begin{array}{l}\text { ¿A las personas fumadoras se les invita } \\
\text { a que acudan a los centros para dejar de } \\
\text { fumar? }\end{array}$ & Revisión de registros & Administración & 1 & 0 & \\
\hline 10 & $\begin{array}{l}\text { ¿Existe un sistema de vigilancia o } \\
\text { supervisión para el cumplimiento de los } \\
\text { programas contra el tabaco? }\end{array}$ & Revisión de documentos & Administración & 5 & 0 & \\
\hline 11 & $\begin{array}{l}\text { ¿Existen mecanismos para denunciar el } \\
\text { incumplimiento del programa? }\end{array}$ & $\begin{array}{l}\text { Revisión de documentos y } \\
\text { procedimientos }\end{array}$ & Administración & 2 & 0 & \\
\hline 12 & ¿Se vende tabaco dentro del edificio? & $\begin{array}{l}\text { Observación directa y } \\
\text { entrevista aleatoria }\end{array}$ & Áreas comunes & 0 & 1 & \\
\hline
\end{tabular}

su visita preguntara directamente para el ítem 3 y 4 ¿Sabe usted si existe algún programa para evitar el consumo de tabaco en el edificio? Con solo dos posibles respuestas sí o no, en caso de ambigüedad, deberá interrogarse a otra persona.

Para lograr una respuesta afirmativa del ítem 8, deberá recorrerse el edificio completo siendo mucho mas acucioso tanto en talleres, cocinas, áreas de maquinas, baños y oficinas de directivos y mandos, donde habitualmente se respetan menos este tipo de programas. Cualquier indicio de uso de tabaco se calificara inmediatamente con cero puntos, por lo que no podrá lograr el reconocimiento.

En caso de que algún edificio no lograse después de haber solicitado su reconocimiento, se le harán las observaciones pertinentes y se visitara nuevamente en un periodo no menor a tres meses. COMO LIBRE DE HUMO DE TABACO, DEBERÁ REA- 


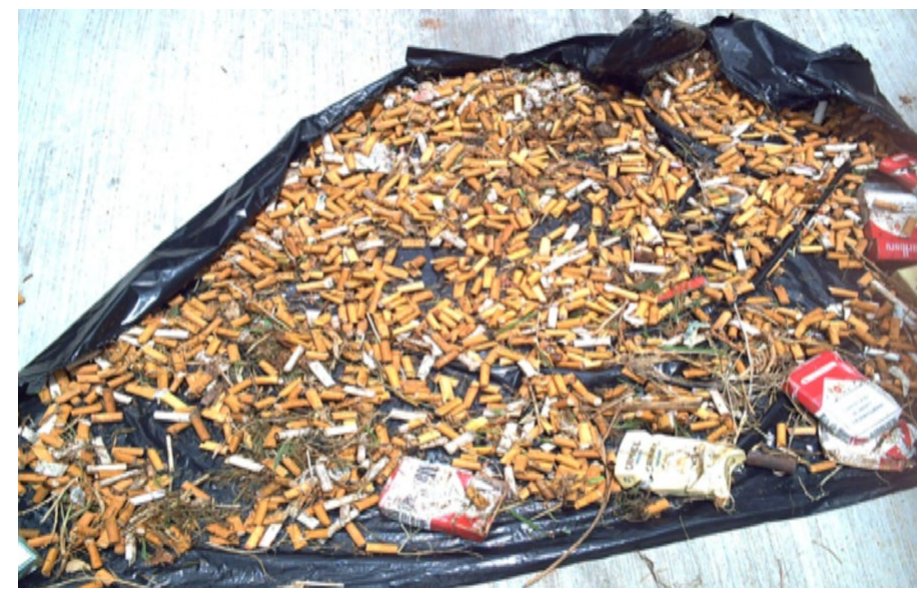

En una de las visitas en un edificio del Campus UAZ Siglo XXI encontraron colillas de cigarro en el frente y se nos solicitó limpiar esto, para volver evaluar y se colecto un recipiente de 8 litros de colillas de tabaco.

LIZARSE UN EVENTO DE SER POSIBLE CON PRESENCIA DEL SECRETARIO DE SALUD Y LA AUTORIDAD MAXIMA DEL ESTABLECIMIENTO, CON LO CUAL OTROS EDIFICIOS E INMUEBLES BUSCARAN LA FORMA DE SER RECONOCIDOS EN BENEFICIO DE SUS TRABAJADORES Y CLIENTES.

3. Que la Comunidad estuviera enterada del proceso.

4. Colocación de ceniceros a la entrada del edificio.

5. Señalamientos de espacio $100 \%$ libre de Humo de Tabaco.

6. Señalamiento del Reglamento de la UAZ como espacio 100\% libre de Humo de Tabaco.

Ejemplo de reglamento, y cada espacio universitario es personalizado.

Reglamento Rectoría.

Reglamento de espacios $100 \%$ libres de Humo de Tabaco de la Rectoría UAZ

\section{DISPOSICIONES GENERALES}

Art. 1.- El presente Reglamento tiene por objetivo la protección de la salud de los no fumadores en Rectoría y así mismo invitar a los fumadores a respetar el edificios de la Administración Central

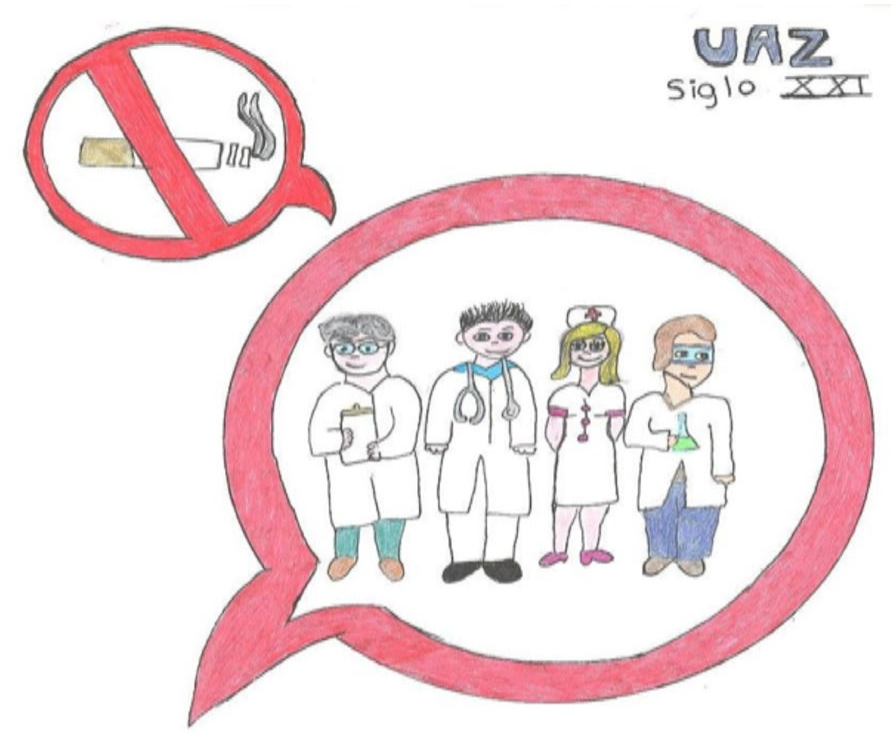

\section{Bos profiesionalles de la Sallud encl confliol dell tabaco}

Figura 3. Publicidad en todos los editicios del Campus UAZ Siglo XXI (Dibujo Claudia Muñoz M. 2007).

como espacios $100 \%$ Libres del humo del tabaco.

Art. 2.- La Protección de la Salud comprende:

A.- El derecho de los no fumadores a no estar expuestos al humo de tabaco en espacios cerrados.

B.- Orientar a todos los integrantes de la Administración Central de los daños del tabaco.

C.- Convocar a todos los integrantes de la Administración Central de la UAZ Siglo XXI que Rectoría sea un espacio $100 \%$ libre del Humo del Tabaco.

D.- Que los integrantes de la Comunidad de la Administración Central tienen el compromiso con la sociedad de no ser adictos al tabaco.

\section{PROGRAMA CONTRA EL TABAQUISMO}

Art 3.- La prevención del tabaquismo tiene carácter prioritario.

A.- La Promoción de la Salud.

B.- Orientación a los no fumadores.

C.- Orientación a los fumadores, para evitar el consumo de tabaco en la escuela, trabajo, hogar y cualquier espacio cerrado y abierto, con el objetivo de cuidar su salud. 


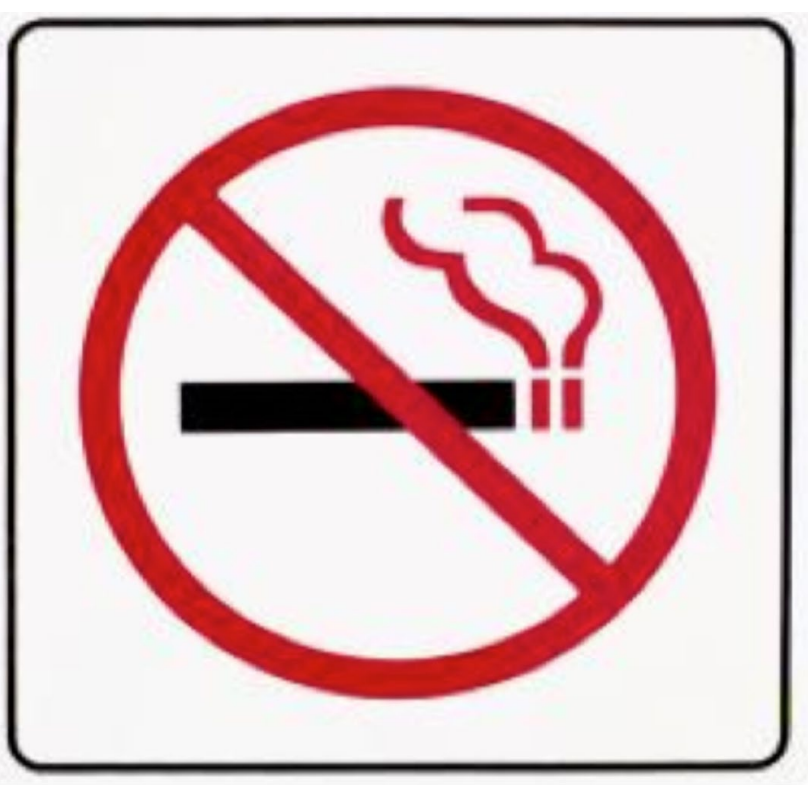

Por favor Apague su Cigarrillo este es un Edificio Libre de Humo de Tabaco

D.- La responsabilidad de la comunidad de la Administración Central hacia la sociedad en la protección de su salud en contra de la adicción del tabaco.

Art 4.- Compromisos de la Universidad Autónoma de Zacatecas.

A.- Compromiso con los Universitarios promocionando en todos los espacios universitarios que sean espacios 100 \% Libres del Humo del Tabaco.

B.- Orientación e información del daño del tabaco.

C.- Servicio informativo a la sociedad sobre la importancia de no fumar.

D.- Compromiso con la comunidad por una Universidad saludable comprometida con su medio ambiente.

\section{Análisis de resultados}

La primera Etapa, se certificó el área de Ciencias de la Salud y CECIUAZ.

En la segunda etapa se certificó 45 espacios de la UAZ (2013).

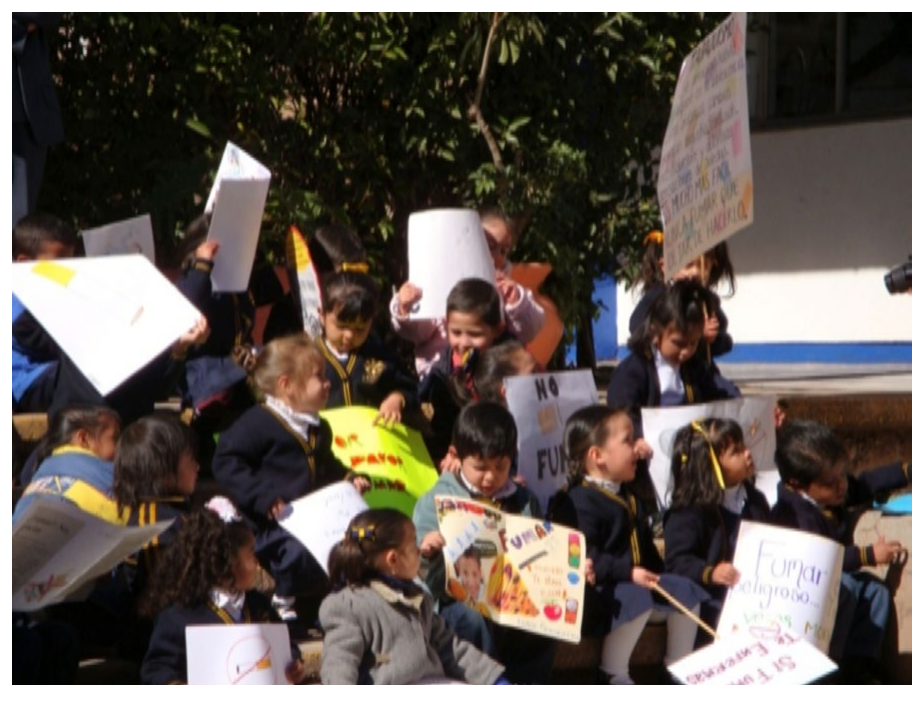

Figura 5. Comunidad CECIUAZ, el día de su reconocimiento como espacio 100\% Libre de Humo de Tabaco.

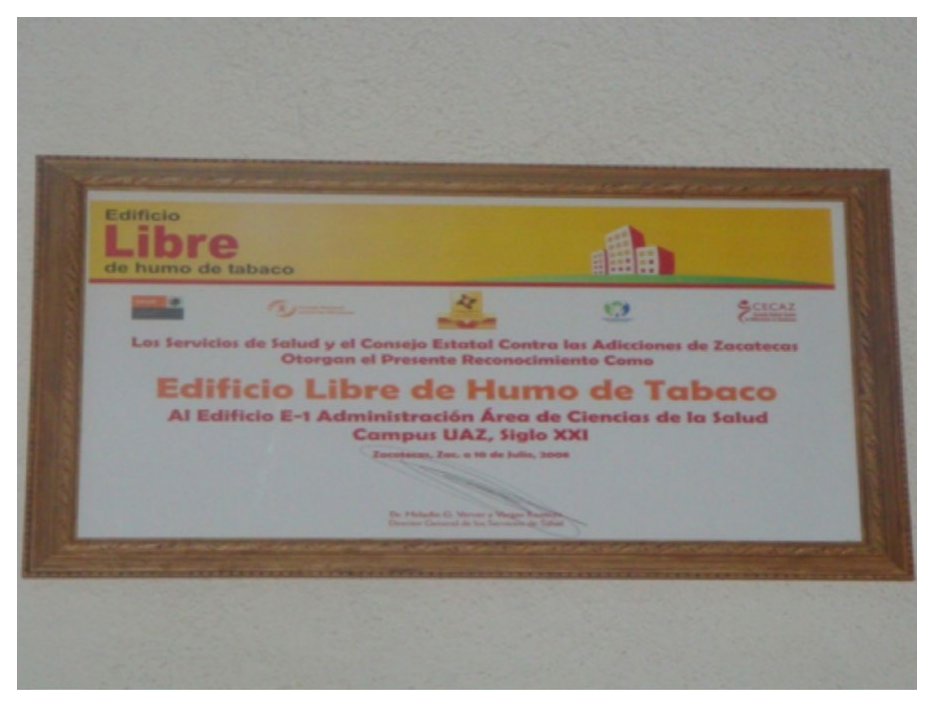

Figura 6. Reconocimiento del edificio E1 del Área de Ciencias de la Salud (2008).

\section{Conclusiones}

Con este reconocimiento inicia el trabajo de retroalimentación y forma parte de la formación integral de nuestros alumnos, y del personal docente, trabajadores y administración central. Se programa una evaluación del programa en dos años. 


\section{Referencias bibliográficas}

Convenio marco de la OMS para el control del tabaco. http://www.who.int/fctc/signatories_parties/es/

Eriksen, M., Mackay, J. y Ross, H. (2012). The Tobacco Atlas, Fourth Edition. Atlanta: American Cancer Society and World Lung Foundation.

"ESTADÍSTICAS A PROPÓSITO DEL DÍA MUNDIAL SIN TABACO"

DATOS NACIONALES. INEGI. 31 de mayo de 2013. http://consulta. mx/web/images/Otros\%20estudios/2013/tabaco0.pdf

Froján, M. y Becoña, E. (1999). El hábito de fumar y su tratamiento, revisión de los avances en los últimos diez años de investigación. Revista de Psicología General y aplicada, 52 (4), 463-476.

Ley General para el Control del Tabaco. Aprobada por el Senado de la República. México, 4 de marzo de 2008.

Ley de Protección a la Salud de los No Fumadores en el Distrito Federal. Aprobada por la Asamblea Legislativa del Distrito Federal el 26 de febrero y publicada en la Gaceta Oficial del Distrito Federal el 4 de marzo de 2008, México, 2008.

Ley General para el control del tabaco. 2010. www.diputados.gob. mx/LeyesBiblio/pdf/LGCT.pdf

Medline. (2012). Riesgos del tabaco. Recuperado el 23 de abril de 2013, de: http://www.nlm.nih.gov/medlineplus/spanish/ency/ esp_imagepages/9940.htm

Página de la Organización Mundial de la Salud. Consultada. 15 de Abril de 2013. www.who.int/es/ -

Quesada, M.; Carreras, J.M y Sánchez, L. (2002). Recaída en el abandono del consumo de tabaco: una revisión. Adicciones, Vol.14 (1). págs. 65-78.

\section{Opina sobre este artículo:}
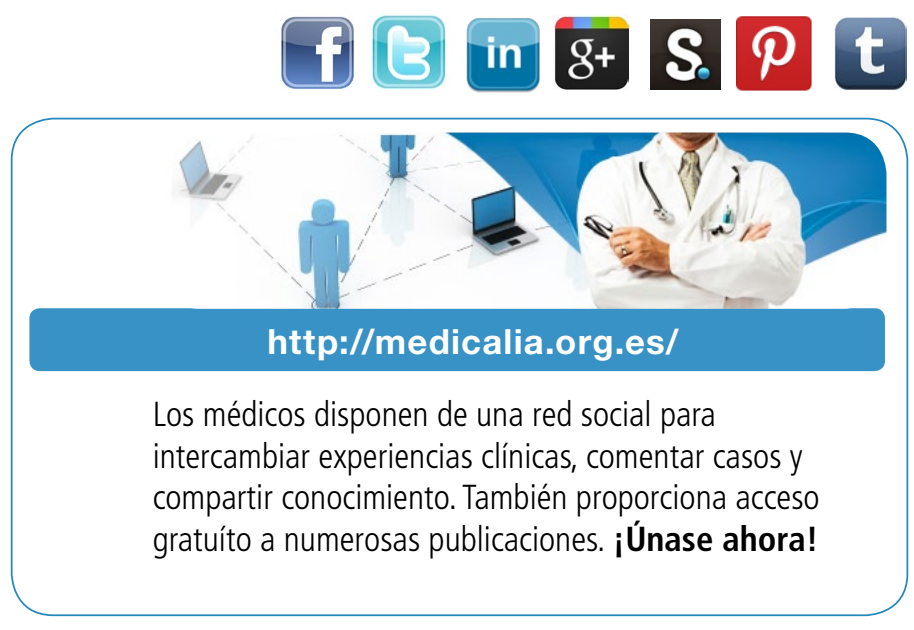

\section{Publique con iMedPub}

http://www.imed.pub

\section{Biomedicina}

$\checkmark$ Biomedicina es la primera revista de acceso abierto en Español, dedicada a todas las áreas de la medicina.

$\checkmark$ Recoge artículos de investigación sobre todas las áreas de la medicina. 